\title{
The Kiss of Death: A New Model for How Perforin Delivers Granzymes to Target Cells Judy Lieberman*‡
}

\author{
Address: CBR Institute for Biomedical Research, Harvard Medical School, Boston, MA, USA \\ Email: Judy Lieberman* - lieberman@cbr.med.harvard.edu \\ * Corresponding author $\ddagger$ Presenting author
}

from 2005 International Meeting of The Institute of Human Virology

Baltimore, USA, 29 August - 2 September 2005

Published: 8 December 2005

Retrovirology 2005, 2(SuppI I):SI06 doi:I0.I I86/1742-4690-2-SI-SI06

Cytotoxic T lymphocytes (CTL) induce target cell apoptosis when they release perforin (PFN) and granzymes (Gzm) into the immune synapse. PFN delivers Gzms into the target cell, but how PFN does this has been unclear. Because PFN forms pores in the plasma membrane, Gzms were originally thought to enter target cells via these pores. However, these pores were too small to allow even small dyes to disseminate into the target. Here we find that PFN dramatically perturbs the target cell membrane, creating pores that transiently allow $\mathrm{Ca}^{++}$and small dyes into the cell. However, the membrane is rapidly resealed and the dyes are circumscribed within membrane proximal blebs. The $\mathrm{Ca}^{++}$flux triggers a wounded membrane repair response in which internal vesicles, including lysosomes and endosomes, donate their membranes to reseal the damaged membrane. The target cell actively participates in determining its own fate during cell-mediated death. The target cell membrane repair response is necessary for target cells subjected to CTL attack to avoid necrosis and undergo the slower process of programmed cell death. However, Gzms do not pass into the cell via PFN plasma membrane pores. Instead PFN triggers the rapid endocytosis of Gzms into large EEA-1-staining vesicles, which then release their cargo into the cytosol to trigger apoptosis. 